\title{
The Effect of Music Therapy on Chronic Pain, Quality of Life, and Quality of Sleep in Adolescents after Transthoracic Occlusion of Ventricular Septal Defects
}

\author{
Ze-Wei Lin, MD, ${ }^{1,2,3,4}$ Jian-Feng Liu, MD, ${ }^{1,2,3,4}$ Wen-Peng Xie, MD, ${ }^{1,2,3,4}$ Qiang Chen, MD, ${ }^{1,2,3,4,5 *}$ \\ Hua Cao, $\mathrm{MD}^{1,2,3,4 *}$ \\ ${ }^{1}$ Department of Cardiac Surgery, Fujian Maternity and Child Health Hospital, Affiliated Hospital of Fujian Medical University, Fuzhou, China \\ ${ }^{2}$ Fujian Key Laboratory of Women and Children's Critical Diseases Research, Fujian Maternity and Child Health Hospital, Fuzhou, China \\ ${ }^{3}$ Fujian Branch of Shanghai Children's Medical Center Affiliated to Shanghai Jiaotong University School of Medicine, Fuzhou, China \\ ${ }^{4}$ Fujian Children's Hospital, Fuzhou, China \\ ${ }^{5}$ Department of Cardiovascular Surgery, Union Hospital, Fujian Medical University, Fuzhou, China
}

\section{ABSTRACT}

Objective: To investigate the effect of music therapy on chronic pain, quality of life, and quality of sleep in adolescent patients after transthoracic occlusion of ventricular septal defects.

Methods: Patients were divided into 2 groups based on whether they received music therapy: a control group and a music group. The music group received 30 minutes of music therapy every day for 6 months after surgery. Patients in the control group received standard treatment and had 30 minutes of quiet time every day for 6 months after surgery. The shortform McGill pain questionnaire (SF-MPQ), the SF-36 scale and the Karolinska Sleep Questionnaire (KSQ) was used as the evaluation tool for chronic pain, quality of life, and quality of sleep, respectively.

Results: In terms of the degree of postoperative chronic pain, the Pain Rating Index (PRI) emotion item score in the SF-MPQ evaluation of the music group was significantly lower than that of the control group $(1.6 \pm 1.1$ versus 2.2 \pm 0.9 ). The role emotional (RE) scores of the SF-36 in the music group were significantly higher than that in the control group $(77.35 \pm 18.55$ versus $42.66 \pm 22.63)$. KSQ scores were significantly higher in the music group than in the control group for sleep status $(4.1 \pm 1.0$ versus $3.3 \pm 0.9)$, falling asleep $(3.9 \pm 1.1$ versus $3.1 \pm 1.0)$, and not feeling refreshed by sleep $(3.6 \pm 1.3$ versus $2.7 \pm 0.9)(\mathrm{P}<.05)$.

Conclusion: This study preliminarily showed that music therapy could effectively reduce patients' chronic pain and improve quality of life and sleep after surgery. These results suggest that music therapy may be an essential therapy worth considering in managing patients' postoperative recovery after cardiovascular surgery.

Received December 6, 2020; received in revised form fanuary 18, 2021; accepted Fanuary 18, 2021.

*These authors share the corresponding authorship.

Correspondence: Hua Cao, MD, Fujian Maternity and Child Health Hospital, Affiliated Hospital of Fujian Medical University, Fuzhou, China (e-mail: caobua0791@163.com).

\section{BACKGROUND}

Ventricular septal defects (VSDs) are common congenital heart malformations [Gan 2008], and sternotomy is the traditional method of treating VSDs. This treatment method is considered the gold standard for most VSDs [Yin 2014], and it is still the first-line treatment for VSDs. However, it has some disadvantages, such as slow recovery and substantial trauma after the operation [Carminati 2007]. With the continuous development of technology, people are satisfied with the same clinical efficacy and have higher requirements for surgical incisions. Ultrasound-guided transthoracic VSD occlusion overcomes the above shortcomings. It has the advantages of a small incision, has no need for cardiopulmonary bypass, and avoidance radiation exposure. Especially in China, it has attracted an increasing amount of attention and achieved good clinical effects [Li 2010; Hijazi 2002]. However, patients often feel postoperative pain due to a variety of factors [van Gulik 2011]. Chronic pain may accompany the patient for a long time or even for life.

Music therapy is widely used in the medical field and has been proven to reduce patients' anxiety and pain [Nilsson 2008]. Through a literature search, there are still few studies on whether music therapy affects the chronic pain, quality of life, and sleep quality of adolescent patients after transthoracic occlusion of VSDs. We designed this study to guide adolescent patients' home care after transthoracic occlusion of VSDs. We hypothesized that music therapy can alleviate adolescent patients' chronic pain, improve quality of life, and improve sleep quality.

\section{METHODS}

This study selected VSD patients admitted from January 2020 to October 2020 as the research subjects. The inclusion criteria were as follows: (1) diagnosis of a single VSD after ultrasound examination; (2) VSD with abnormal hemodynamics, $2 \mathrm{~mm}<$ VSD diameter $<8 \mathrm{~mm}$; and (3) no family history of VSD. The exclusion criteria were as follows: (1) co-occurring congenital heart disease; (2) multiple VSDs; (3) severe insufficiency of other organs; or (4) contraindication 
to surgery. A total of 50 VSD patients were included in this study, and the children were randomly divided into a music group and a control group. There were no statistically significant differences in sex, age, weight, height, VSD diameter, or educational background between the 2 groups $(P>$ $.05)$. Patient demographic data are recorded in Table 1 . The hospital ethics committee approved this study, and all patient guardians signed an informed consent form.

\section{Calculation of Study Sample Size}

PASS15.0 (NCSS, Kaysville, UT) was used to determine the optimal sample size. Assuming that the $\alpha$ value was set at 0.05 with a power of 0.90 , the required number of participants in each group was 22 . Assuming a $15 \%$ missing rate, the total sample size was set as 50 . The patients were divided into 2 groups using a simple randomization method, and a number $(0$ or 1) was randomly generated by a computer. Patients who generated 0 were assigned to the music group $(n=25)$, and patients who generated 1 were assigned to the control group $(n=25)$.

\section{Music Therapy}

The patients chose a quiet room between 8 and 10 o'clock every night and took a comfortable position to lie in bed to rest. They chose one of several kinds of music works provided by the researcher, including but not limited to light music, folk songs, opera, and pop music. Speakers or headphones were used to listen to music for 30 minutes. The music was soft and soothing, and volume control was appropriate. The researchers instructed patients and their families on this method. After being discharged from the hospital, the patients could keep in touch with the researcher on WeChat if they had any questions [Özer 2013].

\section{Control Group}

Patients assigned to the control group chose a quiet room environment between 8 and 10 o'clock every night and had
30 minutes of quiet rest time. The researchers instructed patients and their families on this method. During the followup period, the patients and their families kept in touch with the researchers on WeChat.

\section{Follow-Up}

Echocardiography

After 6 months, each patient was followed up in the clinic to review transthoracic echocardiography results for patients' left atrial diameter (LAD), right ventricular diameter (RVD), left ventricular end diastolic diameter (LVEED), and left ventricular end systolic diameter (LVESD).

\section{Simplified McGill Pain Questionnaire}

We used the standard Chinese version of the simplified McGill Pain Questionnaire (short-form McGill pain questionnaire [SF-MPQ]) [Melzack 1987] to evaluate patients' postoperative pain 6 months after surgery. The SF-MPQ is a simplified version of the MPQ, which includes 4 categories and 30 groups of pain descriptors. It provides a more comprehensive evaluation of pain intensity from the 4 aspects of sensory, emotion, other related factors, and present pain intensity (PPI). The SF-MPQ consists of only 11 sensory and 4 emotional descriptors for pain and the PPI and visual analog scale (VAS). Descriptors indicate different degrees of pain: 0 , none; 1 , light; 2, medium; and 3, heavy. Because the Pain Rating Index (PRI) or the total PRI can be calculated by classification, the SF-MPQ is suitable when the detection time is limited, and other pain intensity information, such as the VAS, is required. Similar to the MPQ, the SF-MPQ is a sensitive, reliable pain evaluation method. Lower scores indicate less severe pain.

\section{6-Item Short Form Survey (SF-36)}

The standard Chinese version of the SF-36 [Ware 2000] was used 6 months postoperatively to assess patients' quality

Table 1. Baseline Characteristics*

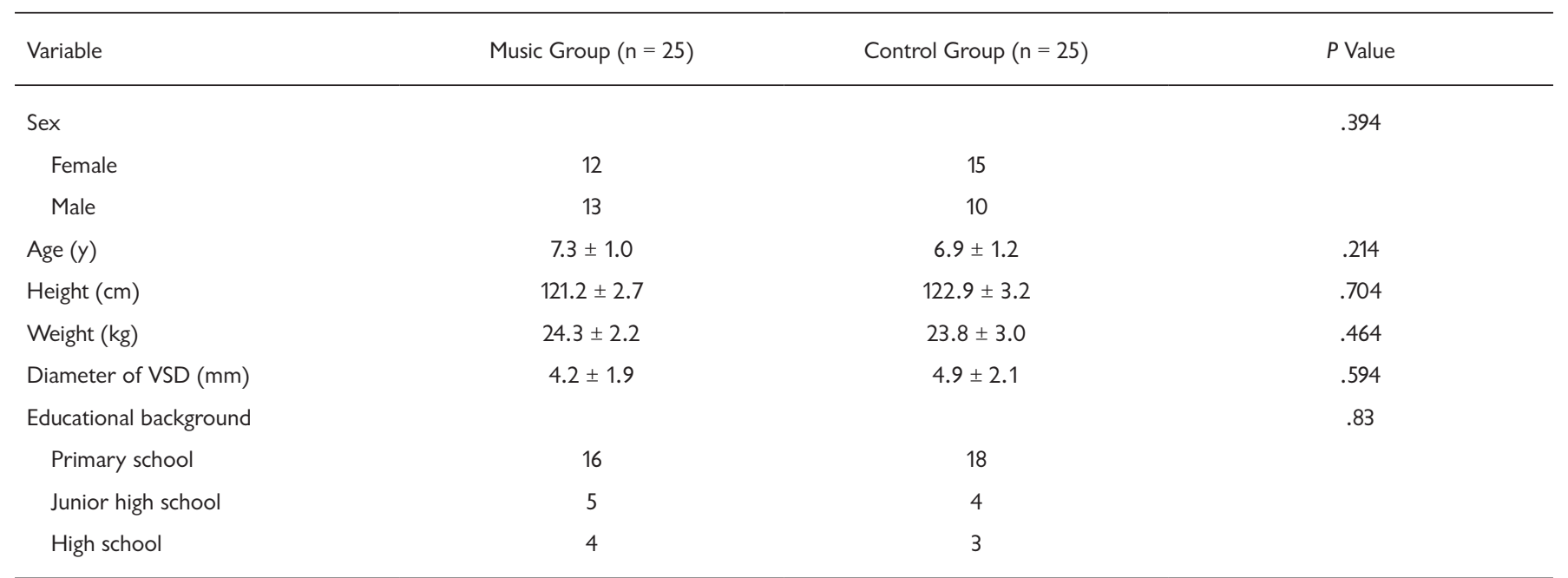

*Data are $n$ or mean \pm SD. 
of life. The SF-36 was developed based on the Mean Opinion Score scale that was developed by Stewartse at the Boston Institute of Health. It is mainly focused on 8 different dimensions: physical functioning (PF), role-physical (RP), bodily pain (BP), general health (GH), vitality (VT), social functioning (SF), role-emotional (RE), and mental health (MH). Different questions explore each dimension, and each question corresponds to a response at a different frequency level and a corresponding score. Respondents completed the questionnaire independently. Each dimension of the SF-36 ranges from 0 to 100 points. Higher scores indicate more favorable conditions.

\section{Karolinska Sleep Questionnaire}

The Karolinska Sleep Questionnaire (KSQ) was used to evaluate sleep quality [Miley 2016]. The scale information was collected at 6 months of postoperative follow-up. Kecklund and Akerstedt developed the KSQ to assess habitual sleep quality through 10 items in 3 dimensions. The first dimension is sleep status (1 item), which is evaluated on a 5 -point scale, ranging from 1 (very poor) to 5 (very good). The second dimension assesses falling asleep, disturbed sleep, difficulty falling asleep again after nocturnal awakening, and early morning awakening (4 items); each item is evaluated on a 5 -point scale, ranging from 1 (every day) to 5 (never). The third dimension assesses daytime distress, including difficulty waking up in the morning, not feeling refreshed by sleep, mental tiredness, exhaustion, and daytime sleepiness (5 items); each item is evaluated on a 5-point scale, ranging from 1 (always/every day) to 5 (never).

\section{Statistical Analysis}

SPSS 25.0 (IBM, Armonk, NY) was used to analyze the data. Categorical data were expressed as $\mathrm{n}(\%)$ and analyzed using $\chi^{2}$ test; measurement data were expressed as mean \pm standard deviation (SD) and analyzed using $t$ test. The normality of the data was assessed; a nonparametric test was used for data that did not have a normal distribution. The $t$ test was used to analyze the sex, age, weight, ventricular diameter, and cardiac color ultrasound data of the 2 groups. The $\chi^{2}$ test was used to analyze the educational background of the 2 groups. The Mann-Whitney $U$ test was used to analyze the differences in scores on the SF-MPQ, SF-36, and KSQ between groups. $P<.05$ indicates that the difference is statistically significant.

\section{RESULTS}

There was no statistically significant difference in baseline characteristics between the 2 groups of patients $(P>.05)$, as shown in Table 1. At the 6-month follow-up, the 2 groups of patients were followed up with transthoracic echocardiography for $\mathrm{LAD}(21.4 \pm 2.4$ versus $20.9 \pm 2.2)$, RVD $(10.9 \pm 2.3$ versus $11.0 \pm 2.0)$, LVEED $(43.3 \pm 5.0$ versus $44.0 \pm 3.9)$, and LVESD $(32.2 \pm 3.6$ versus $33.0 \pm 3.9)$. There was no statistically significant difference in the follow-up data $(P>.05)$, as shown in Table 2. Postoperative reexamination of transthoracic echocardiography indicated that the 2 groups of patients had good cardiac function recovery.
Table 2. Transthoracic Echocardiography 3 Months After Operation*

\begin{tabular}{lccc}
\hline Variable & Music Group $(\mathrm{n}=25)$ & Control Group $(\mathrm{n}=25)$ & $P$ Value \\
\hline LAD & $21.4 \pm 2.4$ & $20.9 \pm 2.2$ & .467 \\
LVEED & $43.3 \pm 5.0$ & $44.0 \pm 3.9$ & .699 \\
LVESD & $32.2 \pm 3.6$ & $33.0 \pm 3.9$ & .475 \\
RVD & $10.9 \pm 2.3$ & $11.0 \pm 2.0$ & .868 \\
\hline
\end{tabular}

*Data are mean $\pm S D$.

Table 3. SF-MPQ Scores*

\begin{tabular}{lccc}
\hline Item & Music Group $(\mathrm{n}=25)$ & Control Group $(\mathrm{n}=25)$ & $P$ Value \\
\hline PRI (sensory) & $1.1 \pm 0.8$ & $1.0 \pm 0.7$ & .739 \\
PRI (emotional) & $1.6 \pm 1.1$ & $2.2 \pm 0.9$ & .022 \\
VAS & $3.1 \pm 1.5$ & $3.5 \pm 1.5$ & .257 \\
PPI & $1.6 \pm 1.1$ & $1.7 \pm 1.2$ & .621 \\
\hline
\end{tabular}

*Data are mean \pm SD.

Table 4. Postoperative SF-36 Scale Scores*

\begin{tabular}{lccc}
\hline Variable & Music Group $(n=25)$ & Control Group $(n=25)$ & $P$ Value \\
\hline PF & $56.00 \pm 8.78$ & $57.60 \pm 9.14$ & .430 \\
RP & $60.00 \pm 21.65$ & $64.00 \pm 20.51$ & .555 \\
BP & $56.1 \pm 13.85$ & $54.40 \pm 13.56$ & .513 \\
GH & $52.20 \pm 13.31$ & $54.20 \pm 11.96$ & .203 \\
VT & $56.80 \pm 11.17$ & $54.60 \pm 10.20$ & .444 \\
SF & $60.22 \pm 16.60$ & $58.40 \pm 15.83$ & .750 \\
RE & $77.35 \pm 18.55$ & $42.66 \pm 22.63$ & .000 \\
MH & $77.76 \pm 3.07$ & $76.32 \pm 4.46$ & .280 \\
\hline
\end{tabular}

*Data are mean \pm SD.

In the postoperative SF-MPQ score of the music group, the PRI sensation item score was slightly lower than that of the control group, but the difference was not significant. The PRI emotion item score of the music group was significantly lower than that of the control group $(1.6 \pm 1.1$ versus $2.2 \pm$ $0.9)$, as shown in Table 3 . The VAS and PPI scores of the 2 groups of patients were similar. The SF-36 scores showed that the RE scores in the music group were significantly higher than those in the control group $(77.35 \pm 18.55$ versus $42.66 \pm 22.63)$. The comparison of other dimensions were not statistically significant, as shown in Table 4. 
The sleep quality scores of the music group evaluated by the KSQ were significantly higher than those in the control group in sleep status $(4.1 \pm 1.0$ versus $3.3 \pm 0.9)$, falling asleep (3.9 \pm 1.1 versus $3.1 \pm 1.0)$, and not feeling refreshed by sleep $(3.6 \pm 1.3$ versus $2.7 \pm 0.9)$, and the differences were statistically significant $(P<.05)$, as shown in Table 5 .

\section{Availability of Data and Materials}

The datasets used and analyzed during the current study are available from the corresponding author on reasonable request.

\section{DISCUSSION}

VSDs can damage patients' cardiac function, affect the blood and oxygen supply of the heart and its regular operation, and pose a severe threat to patients' lives. Transthoracic occlusion of VSDs is one of the classic surgical methods in the clinic at present. Because of its apparent advantages, it has been widely promoted and applied in China [Kang 2015; Martinez 2019; Zhang 2012]. However, patients inevitably feel pain and anxiety due to concerns about complications. The anxiety of patients often leads to a decline in quality of life. Affected by various factors, such as the patient's physical, emotional, and environmental factors, the patient's sleep quality is significantly reduced [Gültekin 2018]. At present, a large number of clinical articles focus on the methods of surgery and the complications of surgery; there are fewer studies on patients' chronic pain, quality of life, and sleep quality.

As early as in ancient Greece, music was considered to play an important role in medical healing. Its rhythm has a psychological impact and enhances the energy of patients. Music is a nonpharmacological, low-risk intervention with a low simple essential workload, making it an attractive intervention for further study. Music therapy is widely used in medicine, and music therapy can reduce patients' anxiety and pain, improving their sleep [Hole 2015; Vaajoki 2012]. The purpose of this study was to study music's effect on adolescent patients after thoracic closure of VSDs.

After the operation, we reviewed transthoracic echocardiography results during follow-up, including LAD, RVD, LVEED, and LVESD. These indicators were close to the expected standard range. The difference between the 2 groups of patients was not statistically significant, indicating that our selected cases' surgical results were satisfactory.

Pain has always been regarded as an essential factor affecting comfort and recovery after cardiac surgery. Postoperative chronic pain refers to continuous or intermittent pain that lasts for $\geq 3$ months after surgery. Crombie [1998] discussed chronic pain after surgery. Similar to other vital signs, its changes can cause a series of physiological, pathological, and neurobiological changes in the human body. Some patients will feel pain for a long time, and even this feeling will last for a lifetime because of sternum stretching, tissue injury, steel wire friction, and other factors during the operation.

Approximately $30 \%$ of adults in the world suffer from chronic pain. A survey by the World Association of Anesthesiologists shows that $54 \%$ of patients undergoing cardiothoracic surgery still have pain [Lynch 2001], and approximately $10 \%$ of patients will have severe pain [Mercadante 2005]. Approximately $50 \%$ of patients have postoperative pain for 1 to 2 years, and $30 \%$ have postoperative pain for 4 to 5 years.

In this study, our medical staff instructed patients in the music group to listen to music every day for 6 months after the operation, and the degree of chronic pain was measured by the SF-MPQ. The results showed that in the postoperative SF-MPQ score of the music group, the PRI emotion score

Table 5. Scores of Postintervention KSQ*

\begin{tabular}{|c|c|c|c|}
\hline Sleep status & $4.1 \pm 1.0$ & $3.3 \pm 0.9$ & $<.01$ \\
\hline Falling asleep & $3.9 \pm 1.1$ & $3.1 \pm 1.0$ & $<.01$ \\
\hline Disturbed sleep & $3.7 \pm 1.1$ & $3.6 \pm 0.9$ & .615 \\
\hline Early morning awakening & $3.2 \pm 1.2$ & $3.0 \pm 1.0$ & .424 \\
\hline \multicolumn{4}{|l|}{ Daytime distress } \\
\hline Difficulty waking up in the morning & $4.5 \pm 1.1$ & $4.2 \pm 0.9$ & .679 \\
\hline Not feeling refreshed by sleep & $3.6 \pm 1.3$ & $2.7 \pm 0.9$ & .001 \\
\hline Mental tiredness & $4.0 \pm 0.8$ & $3.9 \pm 1.0$ & .765 \\
\hline
\end{tabular}

*Data are mean \pm SD. 
in the music group was significantly lower than that in the control group. The results illustrated that the patients experienced relief from chronic pain after receiving music therapy for a relatively long time. In theory, listening to music releases endorphins and lowers the level of catecholamines [Aalbers 2017]. It had been proven that daily pain can be forgotten owing to music's focus and weakened by continuous music input. Many drug-based interventions to treat pain are accompanied by adverse effects. It was safe to provide soothing music after surgery, which could help reduce the use of painkillers, and it was relatively cheap and easy to implement.

With the transformation of medical models, people have paid increasing attention to whether a treatment method can enable a better quality of life. Quality of life refers to individuals' experience in different cultures and value systems of the state related to their life goals, expectations, standards, and things they care about, including individual physiology, psychology, social function, and secular state. In this study, we used the SF-36 to assess patients' quality of life after surgery. The scale's evaluation items covered all aspects of the definition of quality of life, and its reliability and validity had been widely reported [Koh 2006; Falcoz 2002]. Therefore, the reliability of the research results was relatively high. The SF-36 has been tested and found to be suitable for assessing quality of life after cardiac surgery [Falcoz 2003].

The study of Hilliard [2003] confirmed that the application of music therapy could improve the quality of life of dying patients with painful advanced cancer. Studies had shown that persistent chronic pain after surgery might affect the quality of life score after surgery [Gjeilo 2010]. It could be seen from the SF-36 score, the RE score of the music group in our study was higher than that of the control group. We believed that the application of music in postoperative patients had a significant effect and improved the patient's medium- to long-term quality of life. The improvement of quality of life after surgery helps patients recover physically and provides significant benefits in a faster integration of patients back into society.

Patients' sleep problems may include difficulty falling asleep, reduced sleep time, dissatisfaction with sleep, and decreased sleep quality. Sleep disorders stimulate the sympathetic nervous system and promote excessive secretion of vasoconstrictors, thereby increasing the heart load. Studies had shown that the duration of psychological stress and symptoms such as anxiety and depression could contribute to sleep quality [Kalmbach 2018]. In this study, in the evaluation of sleep quality by the KSQ, the scores of sleep state, falling asleep, and not feeling refreshed by sleep in the music group were higher than those in the control group, a difference that was statistically significant $(\mathrm{P}<.05)$. Good sleep quality plays a vital role in maintaining normal physiological and psychological activities and human health. The higher the score, the better the sleep quality, indicating that postoperative music therapy can divert some attention from postoperative incision pain and negative emotions, improve sleep quality, reduce the burden on the heart, and allow the patient to concentrate more on recovery.

\section{Limitations}

Our research had some limitations. This study's sample size was relatively small and limited to adolescent patients after transthoracic occlusion of VSDs. In addition, this study was not a clinical controlled trial study. Therefore, the results may not be generalizable to other surgical patient groups. To a certain extent, the curative effect of music therapy is also affected by factors such as the inherent content of the music itself, such as rhythm, melody, and timbre, as well as the listener's acceptance level, such as age, education level, etc., which restricted the music to a certain extent of popularization and promotion of therapies. Our data collection may have biases. The data in this study were subjectively affected by patients, and there might be biases in the recording process. We did not study the relationship between chronic pain and postoperative quality of life. We studied the quality of life of patients after surgery. We did not study cultural status, economic level, length of hospital stay, overall expenditure, or other patient factors, and the conclusions have a specific bias. A variety of factors need to be considered in the future, and a larger sample and more extended follow-up study should be conducted to verify our conclusions.

\section{Conclusion}

The preliminary results of this study show that music therapy can be safely and effectively included in the care of adolescent patients after transthoracic occlusion of VSDs, can reduce chronic pain in patients to a certain extent, improve the quality of life of patients after surgery, and improve patients' sleep.

\section{ACKNOWLEDGMENTS}

We gratefully acknowledge the contribution by the participating researchers: Xian-rong Yu, Wang-shen Dai, Ya-li Huang, Qiu-yu Chen, and Li-wen Wang. This study was supported by the National Key Research and Development Program of China (grant no. 2016YFC1301900).

\section{REFERENCES}

Aalbers S, Fusar-Poli L, Freeman RE, Spreen M, Ket JC, Vink AC, Maratos A, Crawford M, Chen XJ, Gold C. Music therapy for depression. Cochrane Database Syst Rev 2017;11:CD004517.

Carminati M, Butera G, Chessa M, De Giovanni J, Fisher G, Gewillig M, Peuster M, Piechaud JF, Santoro G, Sievert H, Spadoni I, Walsh K; Investigators of the European VSD Registry. Transcatheter closure of congenital ventricular septal defects: results of the European Registry. Eur Heart J 2007;28:2361-2368.

Crombie IK, Davies HT, Macrae WA. Cut and thrust: Antecedent surgery and trauma among patients attending a chronic pain clinic. Pain 1998;76:167-171.

Falcoz PE, Chocron S, Mercier M, Puyraveau M, Etievent JP. Comparison of the Nottingham Health Profile and the 36-item health survey questionnaires in cardiac surgery. Ann Thorac Surg 2002;73:1222-1228.

Gan C, An Q, Lin K, Tang H, Lui RC, Tao K, Pan W, Shi Y. Perventricular 
device closure of ventricular septal defects: Six months results in 30 young children. Ann Thorac Surg 2008;86:142-146.

Gjeilo KH, Klepstad P, Wahba A, Lydersen S, Stenseth R. Chronic pain after cardiac surgery: A prospective study. Acta Anaesthesiol Scand 2010;54:70-78.

Gültekin Y, Özçelik Z, Akıncı SB, Yorgancı HK. Evaluation of stressors in intensive care units. Turk J Surg 2018;34:5-8.

Hijazi ZM, Hakim F, Haweleh AA, Madani A, Tarawna W, Hiari A, Cao QL. Catheter closure of perimembranous ventricular septal defects using the new Amplatzer membranous VSD occluder: Initial clinical experience. Catheter Cardiovasc Interv 2002;56:508-515.

Hilliard RE. The effects of music therapy on the quality and length of life of people diagnosed with terminal cancer. J Music Ther 2003;40:113-137.

Hole J, Ball E, Meads C. Music as an aid for postoperative recovery in adults: A systematic review and meta-analysis. Lancet 2015;386:1659-1671.

Kalmbach DA, Anderson JR, Drake CL. The impact of stress on sleep: Pathogenic sleep reactivity as a vulnerability to insomnia and circadian disorders. J Sleep Res 2018;27:e12710.

Kang SL, Tometzki A, Caputo M, Morgan G, Parry A, Martin R. Longerterm outcome of perventricular device closure of muscular ventricular septal defects in children. Catheter Cardiovasc Interv 2015;85:998-1005.

Koh ET, Leong KP, Tsou IY, Lim VH, Pong LY, Chong SY, Seow A; Tan Tock Seng Hospital Rheumatoid Arthritis (TTSH RA) Study Group. The reliability, validity and sensitivity to change of the Chinese version of SF-36 in oriental patients with rheumatoid arthritis. Rheumatology (Oxford) 2006;45:1023-1028.

Li SJ, Zhang H, Sheng XD, Yan J, Deng XC, Chen WD, Hu SS. Intraoperative hybrid cardiac surgery for neonates and young children with congenital heart disease: 5 years of experience. Ann Thorac Cardiovasc Surg 2010;16:406-409.

Lynch M. Pain as the fifth vital sign. J Intraven Nurs 2001;24:85-94.
Martinez JJ, Verma AJ, Thankavel PP, Jaquiss RD. Ventricular pseudoaneurysms after perventricular closure of ventricular septal defect. Ann Thorac Surg 2019;107:e397-e398.

Melzack R. The short-form McGill Pain Questionnaire. Pain 1987;30:191-197.

Mercadante S, Ferrantelli A, Tortorici C, Lo Cascio A, Lo Cicero M, Cutaia I, Parrino I, Casuccio A. Incidence of chronic pain in patients with end-stage renal disease on dialysis. J Pain Symptom Manage 2005;30:302-304.

Miley AÅ, Kecklund G, Akerstedt T. Comparing two versions of the Karolinska Sleepiness Scale (KSS). Sleep Biol Rhythms 2016;14:257-260.

Nilsson U. The anxiety- and pain-reducing effects of music interventions: A systematic review. AORN J 2008;87:780-807.

Özer N, Karaman Özlü Z, Arslan S, Günes N. Effect of music on postoperative pain and physiologic parameters of patients after open heart surgery. Pain Manag Nurs 2013;14:20-28.

Vaajoki A, Pietilä A-M, Kankkunen P, Vehviläinen-Julkunen K. Effects of listening to music on pain intensity and pain distress after surgery: An intervention. J Clin Nurs 2012;21:708-717.

van Gulik L, Janssen LI, Ahlers SJ, Bruins P, Driessen AH, van Boven WJ, van Dongen EP, Knibbe CA. Risk factors for chronic thoracic pain after cardiac surgery via sternotomy. Eur J Cardiothorac Surg 2011;40:1309-1313.

Ware JE Jr. SF-36 health survey update. Spine (Phila Pa 1976) 2000;25:3130-3139.

Yin S, Zhu D, Lin K, An Q. Perventricular device closure of congenital ventricular septal defects. J Card Surg 2014;29:390-400.

Zhang GC, Chen Q, Chen LW, Cao H, Yang LP, Wu XJ, Dai XF, Chen DZ. Transthoracic echocardiographic guidance of minimally invasive perventricular device closure of perimembranous ventricular septal defect without cardiopulmonary bypass: Initial experience. Eur Heart J Cardiovasc Imaging 2012;13:739-744. 